\title{
Two Cases of Cutaneous
}

\section{Angiosarcoma Developed after Breast Cancer Surgery}

\author{
Natsuko Iga Yuichiro Endo Akihiro Fujisawa \\ Yumi Matsumura Kenji Kabashima Miki Tanioka \\ Yoshiki Miyachi \\ Department of Dermatology, Kyoto University Graduate School of Medicine, \\ Kyoto, Japan
}

\section{Key Words}

Angiosarcoma - Breast-conserving therapy - Radiation - Breast cancer - Lymphedema · Stewart-Treves syndrome · Axillary lymph node dissection · Breast cancer surgery

\begin{abstract}
Several randomized trials have shown that breast-conserving therapy (BCT) is as effective as mastectomy and should be a standard treatment for early-stage breast cancer. Recently, there has been an increase in reports of angiosarcoma (AS) after BCT. Herein, we report a case of AS which developed after BCT and a case of Stewart-Treves syndrome with a focus on lymphedema. Chronic lymphedema is the primary risk factor for AS, which was first described in 1948 by Stewart and Treves [Cancer 1948;1:64-81]. Radiation therapy secondarily tends to induce the development of AS, since radiation therapy induces fibrosis and proliferation of lymphatic vessels via cytokines such as vascular endothelial growth factor, which is followed by subclinical chronic edema. It is suggested that axillary lymph node dissection predisposes patients to the development of AS, since it is closely associated with lymphedema. Breast surgeons and radiologists should be aware of skin changes in order to improve the early detection of AS during the follow-up of patients who have undergone $\mathrm{BCT}$, and especially those treated with axillary lymph node dissection.
\end{abstract}

\section{Introduction}

Several randomized trials have demonstrated that breast-conserving surgery followed by radiation therapy, which is called breast-conserving therapy (BCT), is as effective as mastectomy and should be a standard treatment for early-stage breast cancer [1]. Recently, there has been an increase in reports of angiosarcoma (AS) after BCT [2-4]. 
Chronic lymphedema is one of the major risk factors of AS. Lymphedema-associated cutaneous AS, so-called Stewart-Treves syndrome, was first described in 1948 by Stewart and Treves [5]. Herein, we report a case of AS which developed after BCT and a case of Stewart-Treves syndrome with a focus on lymphedema.

\section{Case Report}

Case 1

In 1997, a 62-year-old woman underwent BCT with 50 Gy radiation for left-sided breast cancer, categorized as stage IA. An axillary lymph node dissection exhibited no tumor metastasis. She was started on weekly intravenous paclitaxel at a dose of $100 \mathrm{mg} / \mathrm{m}^{2}$. In May 2009, she presented with an

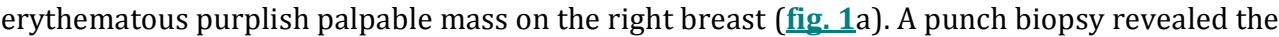
invasion of spindle-shaped cells with vascular differentiation into the subcutaneous tissue (fig. 1b). Immunohistochemistry revealed that these atypical cells were positive for CD31 and CD34. She was diagnosed with AS. A wide local excision was made. Local recurrences were found in December 2009, November 2010, and September 2011. Each time, further wide excisions were performed. Due to these local recurrences, at 85 cycles after the administration of weekly paclitaxel therapy, the patient was switched to monthly docetaxel at a dose of $70 \mathrm{mg} / \mathrm{m}^{2}$. Since then, the patient has not exhibited local recurrence for 7 months.

Case 2

A 64-year-old woman was diagnosed with right-sided breast cancer that was categorized as stage IA. She underwent BCT in August 2007. An axillary lymph node dissection exhibited no tumor metastasis. She received no adjuvant radiotherapy because she had already been treated with chemotherapy using a combination of paclitaxel and carboplatin for ovarian cancer. In November 2011, the patient was admitted to our hospital for evaluation of purplish ecchymosed cutaneous lesions without a palpable mass on the right breast in the setting of chronic lymphedema (fig. 1c). A punch biopsy revealed pleomorphic spindle-shaped cells with little vascular differentiation infiltrated into the dermis (fig. 1d). Immunohistochemistry revealed that these atypical cells were positive for CD31 and CD34. She was diagnosed with AS. A computed tomography scan showed no metastases. The patient was started on monthly docetaxel at a dose of $75 \mathrm{mg} / \mathrm{m}^{2}$. After 1 cycle, she underwent a right simple mastectomy in February 2012. After the surgery, she continued monthly docetaxel. She remained relapse-free at follow-up 4 months after mastectomy.

\section{Discussion}

The estimated incidence of AS after BCT was found to be $0.14 \%$ [4]. Many studies have demonstrated that radiation may be a major risk factor in the development of AS [2-4]. On the other hand, case 2 suggests that chronic lymphedema is the primary risk factor for AS, which was first described in 1948 by Stewart and Treves [5]. Radiation therapy secondarily tends to induce the development of AS, since radiation therapy induces fibrosis and proliferation of lymphatic vessels via cytokines such as vascular endothelial growth factor, which is followed by subclinical chronic edema. It is suggested that axillary lymph node dissection predisposes patients to the development of AS, since it is closely associated with lymphedema.

BCT is equivalent to mastectomy alone in early-stage breast cancer [1]. The increased use of BCT is likely to lead to an increase in the number of patients with AS. The prognosis of AS after BCT is poor, with a low 5-year overall survival (55\%) and a low disease-free survival at 5 years (35\%) [2]. Early detection and treatment is important. Therefore, breast surgeons and radiologists should be aware of skin changes 
in order to improve the early detection of AS during the follow-up of patients who have undergone BCT, and especially those treated with axillary lymph node dissection.
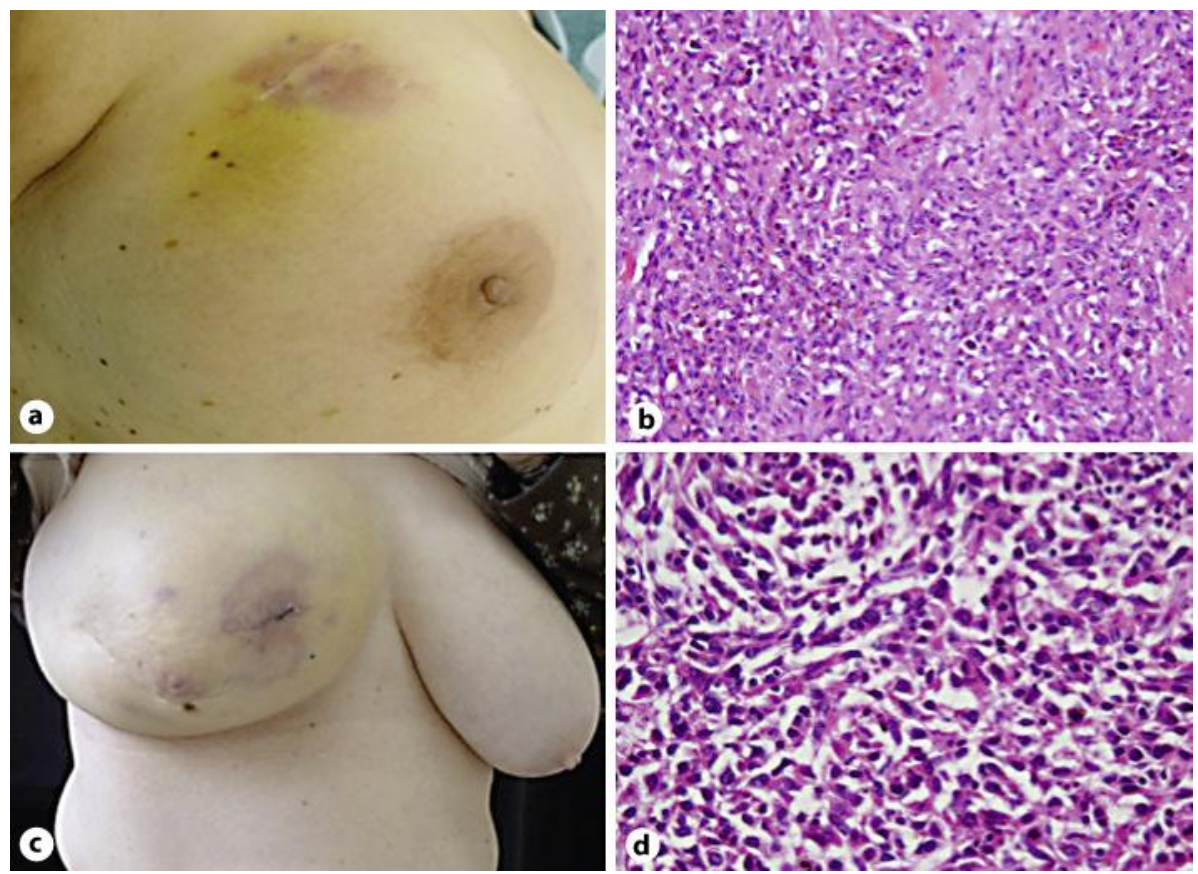

Fig. 1. a Clinical appearance with chronic edema and an erythematous purplish palpable mass on the right breast in case 1. b Histological examination revealed spindle-shaped cells with vascular differentiation. c Clinical appearance with chronic edema and purplish ecchymosed skin lesions on the right breast in case 2 . d Histological examination revealed pleomorphic spindle-shaped cells with little vascular differentiation.

\section{References}

1 Litiere S, Werutsky G, Fentiman IS, Rutgers E, Christiaens MR, Van Limbergen E, et al: Breast conserving therapy versus mastectomy for stage I-II breast cancer: 20 year follow-up of the EORTC 10801 phase 3 randomised trial. Lancet Oncol 2012;13:412-419.

-2 Strobbe LJ, Peterse HL, van Tinteren H, Wijnmaalen A, Rutgers EJ: Angiosarcoma of the breast after conservation therapy for invasive cancer, the incidence and outcome. An unforseen sequela. Breast Cancer Res Treat 1998;47:101-109.

-3 Yap J, Chuba PJ, Thomas R, Aref A, Lucas D, Severson RK, et al: Sarcoma as a second malignancy after treatment for breast cancer. Int J Radiat Oncol Biol Phys 2002;52:1231-1237.

-4 Fodor J, Orosz Z, Szabo E, Sulyok Z, Polgar C, Zaka Z, et al: Angiosarcoma after conservation treatment for breast carcinoma: our experience and a review of the literature. J Am Acad Dermatol 2006;54:499504.

-5 Stewart FW, Treves N: Lymphangiosarcoma in postmastectomy lymphedema; a report of six cases in elephantiasis chirurgica. Cancer 1948;1:64-81. 